\title{
Research on Bulk Cement Logistics Optimization Based on Quantitative \\ Loading System
}

\author{
Feng Guo, Liang Tao, Xia Chen, Jin-Li Li, Fang Wu \\ School of Control Engineering, Northeastern University in Qinhuangdao, Hebei, China \\ E-mail: 1296528878@qq.com
}

\begin{abstract}
In this paper, the quantitative loading and weighing system is designed to optimize the current bulk cement delivery process. Directing at the traffic congestion and reciprocating weighing problem during the cement plant vehicles in and out of the factory, the article designs a quantitative loading system by installing a truck scale in the bulk base bottom, applying the technology of radio frequency identification, vehicle weighing and quantitative loading. Through the designing optimization and the once completed loading, the weighing process of the bulk cement vehicle in the entrance and exit is simplified, which not only reduces the residence time of the vehicle in the factory, but also avoids the loading error caused by the traffic reciprocating.
\end{abstract}

Keywords-RFID; Wireless networks; Bulk cement; Quantitative loading; Import and Export system

\section{INTRODUCTION}

The cement industry is one of the important pillar industries of the national economy, closely related to the development of production and construction, occupying an important position in the national economy[1]. With the development of China's economy, China's cement demand and production are growing rapidly[2]. The realm of sales of cement require more efficient, fast and accurate loading and transportation. Moreover, sacked cement has gradually failed to meet the market demand. The past two decades, bulk cement has developed with great rapidity, as the main form of cement sales, the rate of bulk cement even up to $90 \%$ in some areas.

Currently, cement companies adopt manual loading quantitative way[3], hence the vehicle in and out of the factory need to be weighed twice, retreat more fill less, from several times to achieve accurate loading. There are some management loopholes and risks in the actual operation process, mainly are reflected in the man-made operation, which can not be placed in effective supervision and control. Therefore, it is urgent to propose a new way of loading in order to solve the problems, which exist in manual loading mode, such as slow loading speed, low precision of loading and poor stability. The quantitative loading system[4] is a modern loading mode introduced from abroad at the end of the 20th century and can be real-time monitored on the spot. It features a stable and reliable performance, high accuracy, high efficiency, low environmental pollution and other advantages[5], which is rapidly spread in China's cement enterprise delivery system.
In order to solve the problem of manual loading and improve the efficiency of bulk cement delivery, in this paper, combining quantitative loading system, programmable controller with weighing system[6], applying the technology of radio frequency identification[7], design a quantitative loading system. Consequently, weighing and loading can be completed at one time. In addition, the vehicle at the entrance need not to be weighed, which not only reduces the vehicle's residence time in the factory, but also to avoid the round trip caused by overload. The bulk cement loading efficiency has been greatly improved.

\section{BULK CEMENT IMPORT AND EXPORT SYSTEM}

\section{A. Bulk Cement Radio Frequency Identification System}

Radio frequency identification (RFID) as a kind of advanced automatic identification and data acquisition technology, is recognized as one of the most promising information technology in twenty-first Century, which has been successfully applied to various fields of manufacturing, logistics management, public security and other fields [8].It is based on the identification of objects without contact to obtain identification information of objects. Compared with the traditional identification technology, it has large data capacity, non-contact identification, and it can save time and adapt to the harsh environment, which is widely used in various industries.

Radio frequency identification (RFID) technology is introduced into the bulk cement loading system of cement plant, which can realize the functions of supervision and management, automatic identification, continuous loading and automatic recording. Radio frequency identification system mainly consists of three parts: card reader, electronic tag and computer card reader software. In the loading identification system of bulk cement vehicle in cement plant, the electronic label pasted on the front window of the automobile is collected by the remote card reader installed on the road. After the vehicle carries the electronic tag into the magnetic field, the reader sends out the radio frequency signal, and sends out the data information stored in the electronic label by the energy obtained from the induced current. Then the reader sends the data information to the computer software for decoding, and the vehicle information is retrieved from the database.

Under the traditional operation mode, the drivers need to submit the bill, and then the operators record the information 
according to the bill. However, in the new radio frequency identification system, information can be infinitely scanned and called by recording the vehicle information once at the scene of loading. It not only simplifies the process of submitting and recording, but also improves the degree of safety and the efficiency of loading cement. It solves the problem that drivers should get on or off frequently to hand in tickets, which can shorten the time of loading operation.

\section{B. The Traditional Process of Bulk Cement in and out Factory}

The traditional process of bulk cement in and out factories: the empty vehicle enter the factory, the drivers should check in and receive card, and then they should drive to the truck scale for the first weighing. After they finish it, bound for the feed opening and the field staff in feed opening will operate the equipment to load cement according to the read information. And then drivers should go to the truck scale for the second weighing. They can get the weighing single print and leave factory after contrasting and correcting. The traditional process is shown in figure 1.

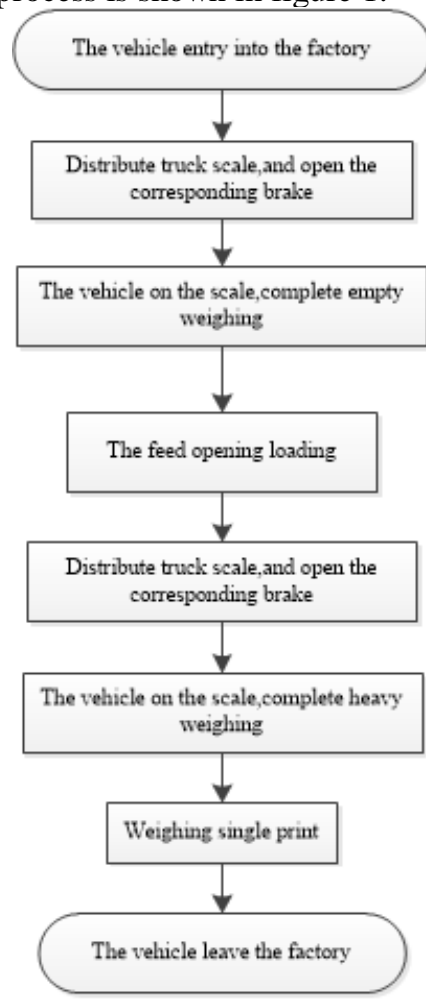

Figure 1. The traditional process of bulk cement in and out of factories

The loading process between traditional bulk cement and bagged cement is almost alike. Since the manufacturing scale of bulk cement is more than the bagged cement, it causes some new problems. The bagged cement weigh of each bag is $50 \mathrm{~kg}$. Hence the total bagged cement weighs of practical and theoretical barely have difference by calculating the number of package. While owing to the bulk cement's quality and weigh are both different, loading by human is quite difficult to realize weighing accurately. In order to reach the target weight, the vehicles may need to round trip several times for adding or reducing cement. It will greatly reduce the efficiency of loading and moving bulk cement. In addition, the process of getting in or out factories exists many vulnerabilities and risks. For example, without permission the vehicles install tanks that water capacity is more than 1t. Before trucks get in factories, drivers will fill up tanks with water. And then they will release water in loading. It will cause loss for companies if this phenomenon is not found.

\section{THE SYSTEM OPTIMIZATION OF BULK CEMENT IN AND OUT FACTORY}

Because the accuracy of manual operation is not high, the vehicles need to round trip several times, which leads to the low loading efficiency. If the automatic loading can be achieved and the weighing steps can be simplified, the efficiency of loading and moving bulk cement will be greatly improved. Based on the above requirements, this paper designs a quantitative loading system for rapid loading, weighing on the scale and precise adding.

Compared to the traditional vehicle scale system, the quantitative loading system contains programmable logic controller (PLC), computer, video surveillance devices, audio system and RFID system. The weigh data and the state of the feeding mouth can be collected, calculated, controlled, saved and managed in the new system. Schematic diagram is shown in figure 2 .

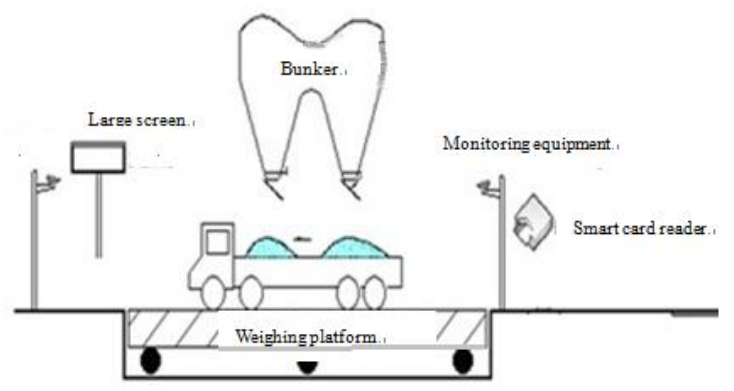

Figure 2. Quantitative loading system

\section{A. Weiging System of Truck Scale}

Truck scales including weighing platform, sensor and instrument, mainly used for tare and net weight for bulk cement vehicle. This paper plans to install a truck scale in the bulk base bottom, once can weigh the empty truck and heavy truck. It can also load weight when in the loading progress, the working process is as follows: when it works, the truck scale is in servo mode, identification of equipment trigger operation by remote card reader. When the vehicle enters the weighing area, the card reader identifies the identity and the truck scale triggers the operation. When the vehicle arrives, the truck scale weight the empty truck, record and send data to the database. After weighting the empty truck, it will trigger quantitative loading system. In the process of quantitative loading, the truck weight is always weighed and displayed. After reaching the preset load, the loading is completed. The truck weight the heavy truck weight, and display and record the data. 


\section{B. Quantitative Loading System}

The quantitative loading system mainly includes computer, programmable logic controller (PLC) and weighing instrument. Run time, the three work at the same time. The computer sends the information to the server and receives the instruction, at the same time forwards the instruction to the PLC, reads the weighing instrument information; the weighing instrument collects the weight information of the automobile scale, and carries on the communication with the computer. PLC main valve to change the discharge speed. In the loading process, the computer communicates with the weighing instrument, weight and calculate the empty truck, loading plan, actual load and the vehicle weight. PLC real-time comparison of current weight and target weight, and shows the current load weight, target weight and weight of the remaining material. These data will be provided to the staff to make decisions. When the weight is far less than the target weight, under the control of PLC outlet valve open to the maximum, it will quickly feed; the current weight calculation system close to the target weight, under the control of PLC outlet valve is small, it will reach the goal of accurate feeding; the weight setting system, under the control of PLC discharge port close the valve, stop loading, loading print ticket. Quantitative loading principle shown in figure 3 .

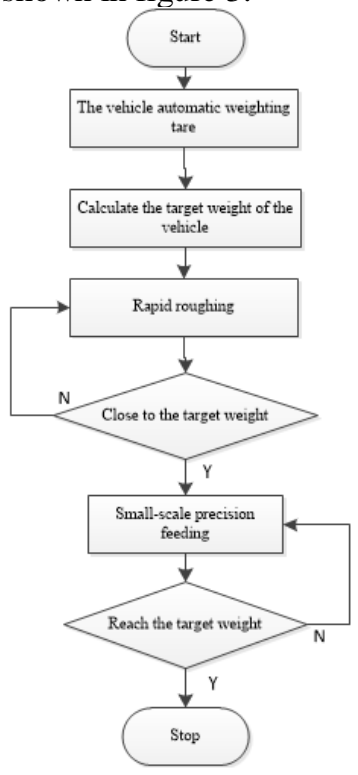

Figure 3. Schematic diagram of quantitative loading

\section{Optimization of Bulk Cement in and out of Factory Process}

The optimized bulk cement factory import process: the empty vehicle into the plant, to guard at registration and receive a card, and then went to feed loading. Automatic scanning vehicle marker for long distance vehicle recognition device, trigger vehicle weighing system. When the vehicle reaches the designated position, automatic quantitative loading weighing, printing inspection report, and then the vehicle can leave factory. The import and export process of bulk cement vehicles is shown in figure 4 .

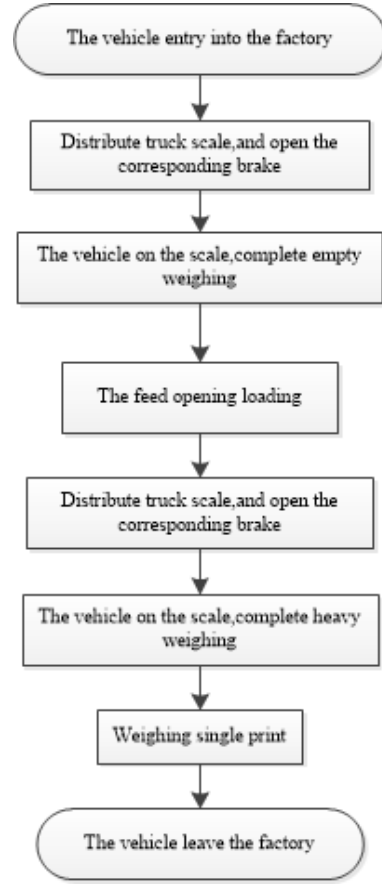

Figure 4. Optimization of bulk cement in and out of the factory process

Compared to the traditional bulk cement in and out of the factory process, the optimization of the quantitative loading weighing system steps less. The bulk cement car only need to drive to the truck scales once, it can complete the entire loading weighing process, consequently it improves the efficiency of loading of bulk cement. The loading process of the real-time display of the current vehicle loading weight, can accurately control the vehicle loading amount of material, to avoid the vehicle load due to too much or too little material loading or unloading caused repeated, reduce the number of vehicles to and from the truck and feed, reduce the cycle time of loading, improve work efficiency.

\section{MAIN FUNCTIONS OF THE SYSTEM}

The quantitative loading and weighing system has realized the function of data collection and calculation of bulk cement truck, the real-time monitoring of the bulk cement vehicle, flexible and diverse report forms, accurate and fast data query and so on.

1) Weighing data collection and calculation. Weighing instrument collects truck weighing data. Computer communicates with weighing instrument and those including empty truck, load capacity, real loading and the whole vehicle weight are calculated and weighed.

2) The control of feeding speed. Always read the total material status, and according to the comparison between the weight and the preset load, PLC sends the instruction to control the feeding speed.

3) Information and image display. Weighing instrument communicates with the display screen to display information 
such as weighing results. Process the video signal and display the real-time image.

4) Perfect the reporting features. Print loading data and count data statistics arbitrarily

\section{CONCLUSION}

Through the optimization of bulk cement loading way, to take effective control of the key link, manual control to automatic control, computer applications to loading link, realize the whole process of intelligent control, which effectively eliminate loopholes in management. Compared with the traditional way of loading, the advantage of the optimized loading way is mainly embodied in the following two aspects: one is a complete weighing and loading, bulk cement vehicle at entrances of truck scale without weighing, which both can reduce the time of vehicles stranded in the factory, and avoid the traffic caused by overload reciprocating to improve the efficiency of the bulk cement delivery. The other is that the link of bulk cement loading is completely controlled, to eliminate possible loopholes and risk in the process of loading, thus safeguard the interests of users and enterprises.

\section{ACKNOWLEDGMENT}

This research was supported by the Fundamental Research Funds for the Central Universities (N142303014), the Science and Technology Research Project of Hebei Province (Z2014085), the Doctoral Scientific Research
Foundation of Northeastern University at Qinhuangdao (XNB201411), Science and Technology Innovation Project of Northeastern University at Qinhuangdao.

\section{REFERENCES}

[1] Energy Efficiency Component EU-China. A Reference Book for the Industry[M]. Beijing, 2009.

[2] Ke J, Zheng N, Price L, et al. Potential Energy Savings and CO2 Emissions Reduction of China's Cement Industry[J]. Energy Policy, 2012, 45:739-751.

[3] Aniki A O, Mbohwa C, Akinlabi E T. Improvement of Logistics and Supply Chain Management in the Cement Industry in Nigeria[J]. Proceedings of the World Congress on Engineering 2014: 280-284.

[4] Yang Z Z. Talking about the Construction of Quick Ration Loading System[J]. Sci-Tech Information Development \& Economy, 2011,21(25):225-226.

[5] Zhang C, Meng L J, Li Y D, et al. Gradient of Loading Lines with Dowty Retarders in Quick Ration Loading System [J]. Sci-Tech China Railway Science, 2014,35(5):121-126.

[6] Hui-Mei He, Pingjie Huang, Dibo Hou, et al. An Intelligent Signal Processing Method for High-Speed Weighing System[J]. International Journal of Food Engineering, 2013, 9(2):179-186.

[7] Jannati Hoda, Ardeshir-Larijani Ebrahim. Detecting Relay Attacks on RFID Communication Systems Using Quantum Bits[J] Quantum Information Processing, 2016,15(11):4759-4771.

[8] Li B, He Y G, Hou Z G, et al. Complementary Metel-oxidesemiconductor Humidity Sensor Design for Passive Ultra-high Frequency Radio-Frequency Identification Application[J]. Acta Phy. Sin, 2014, 63(18):188402. 